\title{
Descripción interpretativa para la elaboración del perfil de tesis de investigación científica con enfoque cualimétrico (mixto)
}

$\begin{array}{r}\text { Interpretative description for the elaboration of the profile of scientific } \\ \text { research thesis with a qualitaimetric approach (mixed) }\end{array}$
Recibido: julio 19 de 2016 | Revisado: agosto 17 d e 2016 | Aceptado: octubre 18 de 2016

Porfirio Enríquez Salas ${ }^{\mathrm{I}}$ George Argota Pérez ${ }^{2}$

1 Universidad Nacional del Altiplano "UNA". Puno, Perú enriquezporfir@yahoo.es

2 Dirección General. Centro de Investigaciones Avanzadas y Formación Superior en Educación, Salud y Medio Ambiente "AMTAWI". Puno, Perú. george.argota@gmail.com

\section{Resumen}

Los formatos de investigación representan modelos de conducción que informan sobre el tipo de investigación a realizar y donde no siempre resultan comprensibles las informaciones consideradas. El objetivo de la investigación fue realizar una descripción interpretativa para la elaboración del perfil de tesis de investigación científica con enfoque cualimétrico. Para ello, se analizó desde enero hasta marzo del 2015, varios formatos relacionados con perfiles de tesis de investigación tanto de ciencias naturales y no afines, procedentes de diversas escuelas de posgrados en universidades del Perú. Se observó que no se detallan, metodológicamente, las informaciones para cada una de las estructuras que conforman los formatos de perfiles de tesis, donde ninguno presentó enfoque mixto de investigación, por lo que, en este trabajo, se describieron informaciones metodológicas que contribuyeron a la interpretación sobre cómo elaborar de forma completa los perfiles de tesis. Se concluyó que la descripción interpretativa para la elaboración del perfil de tesis de investigación científica con enfoque mixto resultó de gran beneficio, pues ello permitió que se pueda dimensionar el patrón de comportamiento sobre el fenómeno, suceso, proceso o evento observado y donde es susceptible, finalmente, efectuar la investigación.

Palabras claves: descripción, elaboración, investigación, perfil de tesis, enfoque cualimétrico o mixto

\footnotetext{
Abstract

The research formats represent driving models that inform about the type of research to be performed and where not always, the information considered is understandable. The objective of the research was to perform an interpretative description for the development of the profile of a scientific research thesis with a qualitative approach. In order to do this, from January to March 2015, several formats related to research thesis profiles of both natural and unrelated sciences from various postgraduate schools at universities in Peru were analyzed. It was observed that the information for each one of the structures that make up the thesis profile formats was not detailed methodologically, where none presented a mixed research approach, so that, in this work, methodological information was described that contributed to the Interpretation on how to complete the thesis profiles. It was concluded that the interpretative
} 
description for the elaboration of the thesis profile of scientific research with a mixed approach carried great benefits, because it allowed to be able to dimension the behavioral pattern on the phenomenon, event or process observed and where it is susceptible, finally, investigate.

Keywords: description, elaboration, research, thesis profile, qualimetric or mixed approach

\section{Introducción}

Ortiz y García (2005) mencionan que pensar en el método científico como un conjunto de normas que constituyen patrones para arribar a determinadas conclusiones como aparentes verdades, solo es posible si las investigaciones estimadas han sido dirigidas responsablemente.

Por su parte, Ruiz (2007) indica que el método científico es el procedimiento planteado que se sigue en la investigación para descubrir las formas de existencia de los procesos objetivos, desentrañar sus conexiones internas y externas, generalizar y profundizar en los conocimientos adquiridos, llegar a demostraciones con rigor racional, comprobar hechos mediante determinados experimentos, así como utilizar técnicas adecuadas que justifiquen la observación.

Considerando lo expresado, según $\mathrm{Na}$ makforoosh (2005) mostró que se hace necesario planificar todo el proceso de la investigación; es decir, desde su elaboración, indicación de etapas a realizar, definir qué es lo que se pretende hacer, tipo de investigación a realizar, datos que se recopilarán y cómo obtenerlo, métodos y técnicas a utilizarse que permitan sustentar dichos datos, siendo necesario dilucidar el tiempo y costo demandado por cada etapa que garanticen la validez de los estudios.
Es por ello que, debido a la creciente necesidad y exigencia por parte de la comunidad científica de garantizar la validez de los diferentes tipos de estudios, se han analizado y creado, constantemente, guías prácticas y recomendaciones sobre cómo establecerla, así como determinar, objetivamente, si un estudio está bien estructurado (Barrientos et al., 2007; Vara, 2012). Este ámbito, que antes se creía correspondía solo a los estudios de tipo cuantitativo, es también una preocupación en el área de investigación cualitativa; y aunque hay diferentes corrientes y opiniones acerca de cómo evaluar este criterio, es evidente que por la diferente naturaleza de la investigación cualitativa con respecto a la cuantitativa, no se deben ni se pueden utilizar los mismos parámetros para su estudio (Cornejo \& Salas, 2011; Okuda \& Gómez 2005).

Artigas y Robles (2010) hicieron una referencia sobre los enfoques cualitativos y cuantitativos, concluyendo que es necesario educar a los investigadores en relación con la combinación de enfoques para que el seguimiento de un determinado esquema, no imposibilite las innovaciones en la búsqueda sobre el mejoramiento del proceso investigativo donde pudiera observarse finalmente, falta de conocimiento a la hora de construir un determinado silogismo.

Grinnell (1997) señala que en términos generales, tanto el enfoque cuantitativo 
como cualitativo, utilizan cuatro fases similares:

1. Ambos enfoques logran probar y demostrar el grado en que las suposiciones o ideas tienen fundamento, a la vez que revisan tales suposiciones o ideas sobre la base de las pruebas o del análisis.

2. Proponen nuevas observaciones para esclarecer, modificar o fundamentar las suposiciones; o incluso para generar otras.

3. En los dos se da la observación y evaluación de fenómenos.

4. Se establecen suposiciones como resultado de la observación y evaluación realizadas.

De manera que es un hecho que ambos enfoques comparten esas etapas generales; sin embargo, cada uno tiene sus propias características. El enfoque cualitativo, por lo general, se utiliza en un primer orden, para descubrir preguntas de investigación. En muchos casos, pero no necesariamente, se prueban hipótesis o proposiciones teóricas. Con frecuencia se basa en métodos de recolección de datos sin medición numérica como las observaciones y las descripciones. En una gran mayoría de ocasiones, las preguntas e hipótesis surgen como parte del proceso de investigación y este es flexible, transitando entre los eventos y su interpretación o entre las respuestas y el desarrollo de las teorías.

El propósito de esta metodología consiste en reconstruir la realidad, tal y como la observan los autores de un sistema social que ha sido previamente definido. Por otra parte, el enfoque cuantitativo utiliza la recolección y el análisis de datos para contestar preguntas de investigación y probar hipótesis que han sido establecidas previamente, la mayor confianza la tiene, en la medición numérica y el uso de la estadística para establecer con exactitud patrones de comportamiento en una determinada población (Grinnell, 1997; Miles \& Huberman, 1994; Stoecker, 1991).

Hernández, Fernández y Baptista (2003) sostienen que ambos enfoques resultan muy valiosos y han realizado notables aportaciones al avance del conocimiento. Ninguno es intrínsecamente mejor que el otro y cada uno sirve a una función específica para conocer un fenómeno, así como conducir hacia la solución de los diversos problemas y cuestionamientos.

Considerando los criterios de los autores anteriores, entonces podría aludirse una pregunta: ¿por qué existen diversos formatos de investigaciones que en múltiples casos, constituyen obstáculos para el desarrollo del propio proceso investigativo?

Es por ello que Argota y Iannacone (2015) señalaron que podría esperarse que la creación de formatos divergentes sobre metodología de la investigación, sin duda alguna, conduciría a diversas consecuencias negativas en un periodo corto de tiempo; ya que si bien puede ser cierto que se interpreten mayores concepciones metodológicas incluidas, realmente será todo lo contrario, pues ello solo implicará que se limite la aplicación del método científico, generándose así reticencia en opiniones científicas, precaria educación científica formativa, acrecentamiento de posibles monopolios del conocimiento científico y tecnológico, distorsión sobre la razón aprehensiva de discernimiento interactivo, así como pérdida del sentido ecuménico de la propia ciencia. Solo puede entenderse la divergencia como respuesta debidamente esperada con relación a las múltiples posibilidades de solución para eliminar las causas y transformar los efectos negativos que conciben un estado actual diferente al deseado como lo es la identificación del problema científico, por lo que resultará la mejor decisión, únicamente pensar en la convergencia 
sobre los formatos en metodología de la investigación científica.

Al utilizar el enfoque cualimétrico (mixto), por ende se entremezclan la perspectiva cuantitativa y cualitativa en la mayoría de sus etapas, de manera que se hace conveniente combinarlos para obtener información que permita la triangulación. Esta triangulación aparece como alternativa de tener la posibilidad para encontrar diferentes caminos y conducirse a una interpretación lo más amplia posible del fenómeno en estudio. El enfoque mixto es un proceso que recolecta, analiza y vincula datos cuantitativos y cualitativos en un mismo estudio o una serie de investigaciones para responder a un planteamiento (Ruiz, Borboa \& Rodríguez, 2013).

En la publicación de Argota y Iannacone (2015) sobre la significación de formatos metodológicos como guías de aprendizaje para la investigación científica de posgrado en ciencias naturales y no afines de universidades peruanas, se mencionan informaciones metodológicas que deben ser consideradas tanto en los perfiles como en el propio proyecto de investigación científica, aunque no se describen los detalles propiamente de dichas informaciones metodológicas.

En tal sentido, y a consideración de los autores, puede indicarse que una de las limitaciones, en muchos postulantes, de programas de formación de posgrado para alcanzar determinados títulos y/o grados, radica exactamente en la falta de comprensión sobre qué debe ser considerado en cada perfil de tesis, estimando sin duda alguna, que cualquier tipo de formato representa más del 75 por ciento de la información a considerar para cualquier proyecto de tesis de investigación científica. El objetivo de la presente investigación fue realizar una descripción interpretativa para la elaboración del perfil de tesis de investigación científica con enfoque mixto.

\section{Métodos}

\section{Objeto de investigación y periodo de es- tudio}

Se analizaron universidades nacionales y privadas del Perú, durante los meses de enero a marzo del 2015.

\section{Población y muestra}

Del total de universidades reconocidas por la Superintendencia Nacional de Educación Superior Universitaria (SUNEDU), fueron seleccionadas mediante un muestreo no probabilístico por conveniencia, siete universidades tanto nacionales como privadas. Sobre las catorce universidades, el método de investigación utilizado fue el cuantitativo empírico por medición.

\section{Análisis de variables}

Se analizó, en cada universidad, las estructuras metodológicas sobre la construcción de enfoques tanto cuantitativo, cualitativo o cualimétrico (mixto) correspondientes a los formatos del perfil de tesis de investigación pertenecientes a sus Escuelas de Posgrado tanto las que poseían una formación hacia las Ciencias Naturales como las no afines.

\section{Análisis de los datos}

Se utilizó como estadígrafo paramétrico descriptivo, el promedio (medida de tendencia central).

\section{Resultados}

La Tabla 1 muestra las 14 universidades seleccionadas, donde se señala su condición como tipo de enfoque de investigación que presentan. 
Tabla 1

Clasificación, condición y enfoque de investigación / universidades seleccionadas

\begin{tabular}{|c|c|c|}
\hline Universidad & Tipo & $\begin{array}{c}\text { Enfoque de } \\
\text { Investigación }\end{array}$ \\
\hline $\begin{array}{c}\text { Universidad Nacional Mayor de San Marcos } \\
\text { (UNMSM) }\end{array}$ & & \\
\hline Federico Villarreal (UNFV) & & \\
\hline San Agustín (UNAS) & Nacional & \\
\hline San Antonio Abad del Cusco (UNSAAC) & & \\
\hline San Cristóbal de Huamanga (UNSCH) & & \\
\hline Del Altiplano (UNAP) & & \\
\hline De Trujillo (UNT) & & \\
\hline Pontificia Católica del Perú (PUCP) & & \\
\hline Cayetano Heredia (UPCH) & & \\
\hline Del Pacífico (UP) & & \\
\hline Ricardo Palma (URP) & & \\
\hline César Vallejo (UCV) & & \\
\hline San Martín de Porres (USMP) & & \\
\hline Alas Peruanas (UAP) & & \\
\hline
\end{tabular}

La Tabla 2 muestra, de forma general, las informaciones que consideran los formatos relacionados con los perfiles de tesis de investigación científica en las universidades (enfoque cuantitativo).

Tabla 2

Informaciones estructuradas de los perfiles de tesis de investigación cientifica

\section{Formato de los Perfiles de Tesis de Investigación Científica}

- Título del proyecto

- Resumen (palabras claves)

- Planteamiento del problema científico

- Justificación de la investigación

- Marco teórico

- Antecedentes

- Hipótesis de investigación

- Objetivos: general (s) y específico (s)

- Materiales y métodos

- (objeto de investigación y periodo de estudio, población y muestra, análisis de variables, análisis de los datos, operacionalización de las variables)

- Cronograma de actividades

- Presupuesto de investigación

- Referencias

- Anexos (matriz de consistencia) 
La Tabla 3 muestra las informaciones que podrán ser consideradas, en los formatos relacionados con los perfiles de tesis de investigación científica con enfoques cualitativos para las universidades.

Tabla 3

Informaciones estructuradas de los perfiles de tesis de investigación cientifica

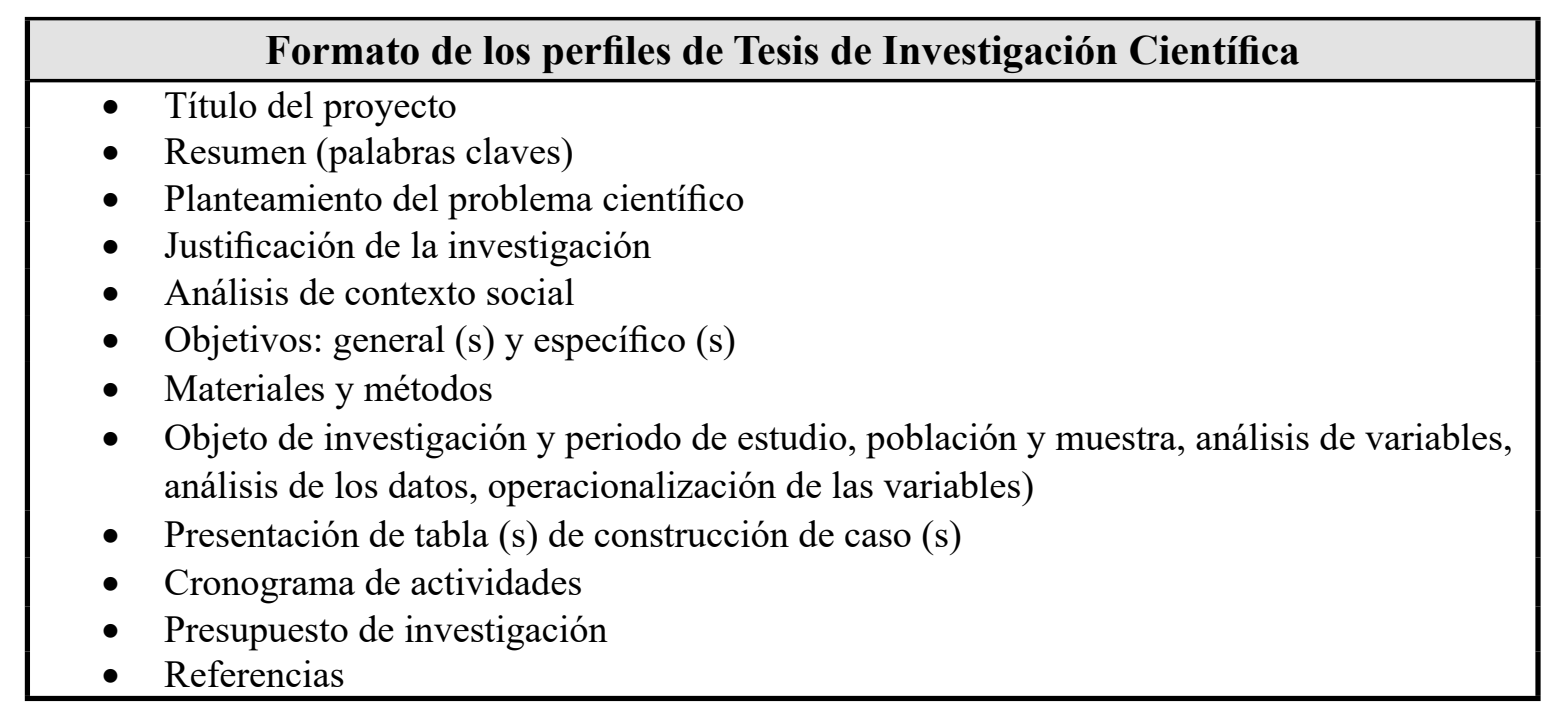

Según Charaja (2011), lo más importante de cualquier proceso sobre investigación científica, es que se demuestre la aplicación del método científico, por cuanto ello puede justificar que formatos relacionados con enfoques cuantitativos como cualitativos, puedan ser utilizados.

A continuación, pueden valorarse las informaciones a considerar por cada estructura metodológica, tanto para los perfiles de investigación con enfoque cuantitativo como cualitativo.

\section{- Título}

El título debe reflejar qué se realizará, cómo se realizará, en qué se realizará, así como dónde se realizará. Existe una tendencia en que los títulos sean cortos y que no superen las 15 palabras, pero ello no debe ser una condición de imposición, pues en muchos casos, podría resultar la expresión con términos de ambigüedad solo por tratar de establecer determinado número de palabras.
Una de las mayores dificultades, en los títulos, es que expresan la realización de una investigación, estudio sobre determinadas situaciones, evitar sobre explicaciones, usos excesivos de preposiciones y artículos, pues si se sobreentiende que es una investigación o estudio, entonces por qué se mencionan. Un título debe demostrar el carácter especializado de la información con relación a la disciplina que se esté abordando, así como denotar una correcta sintaxis. Los títulos no son oraciones y deben finalmente evidenciar según las variables a medir, el tipo de estudio o investigación realizada.

\section{- Resumen}

El resumen es un tipo de instrumento de aprendizaje que puede ser corto o largo según el estado actual de conocimiento que se posea. No necesariamente, un resumen debe comenzar con el anuncio de la situación problemática. Por lo general, dada las tendencias, en muchas revistas científicas indexadas, de admitir hasta un nú- 
mero determinado de palabras, entonces el poder de síntesis no solo es relevante, sino además poder saber estratégicamente cómo comenzar a elaborar determinado resumen. Este debe ser breve, pues en este deben aparecer solo los detalles importantes, las ideas fundamentales y los datos técnicos más sobresalientes. Se debe redactar como un texto normal, a renglón seguido, en uno o varios párrafos, pero sin usar guiones ni sangrar el texto. Todas las ideas deben estar relacionadas entre sí, integradas en un conjunto que les dé unidad y sentido. Un buen resumen podrá estructurarse de la siguiente manera:

1. Breve introducción

2. Objetivo (qué se realizará, cómo y dónde)

3. Tipo de investigación y periodo de ejecución (realización)

4. Tipo de estudio (cuantitativo o cualitativo) y mención de las variables (denominación)

5. Tipo de muestreo (probabilístico o no probabilístico)

6. Tipo de técnica

7. Análisis de las variables (métodos)

8. Técnica analítica de medición de las variables

9. Análisis estadístico de los datos (tipo de software, estadígrafo (s) y nivel de confianza de los datos)

\section{- Palabras claves}

Las palabras claves no son más que los lexemas relacionados con el propio título. Estas palabras no deberán ser alteradas, pues finalmente guardarán relación en orden de aparición una vez concluida la investigación y que se publiquen los resultados. Existe una tendencia en organizar las palabras claves en orden alfabético y aunque esto no es un error, pero podría interpretarse como una carencia de sentido del cómo se hizo inicialmente y luego qué se hizo. Las palabras claves, se redac- tan en letras minúsculas y, por lo general, en cursiva.

\section{- Planteamiento del problema científico}

$\mathrm{Al}$ redactar el problema científico, debe reflejarse la situación problemática, inicialmente, es decir, deberá mostrarse la solución incorrecta o incompleta que ha sido aplicada, pero tendrá que indicarse, de forma cronológica dichas aplicaciones. El nivel de información que se considerará tiene que construirse atendiendo a lo que se requerirá analizar como variables de medición. Una vez terminada de redactar la situación problemática, entonces deberá quedar bien estructurado o formalmente lo siguiente:

1. Importancia del problema práctico

2. Problema práctico concreto

3. Problema general

4. Problema específico

Al redactarse el problema científico, deberá indicarse su planteamiento en forma de interrogante o hipótesis, mostrando la relación entre variables. Finalmente, se deberá indicar la (s) pregunta s) de investigación general (es) y específica (s). Estas preguntas siempre se expresarán en tiempo futuro.

\section{- Justificación de la investigación}

La justificación de la investigación no es más que la razón fundamentada sobre el porqué se realizará. Esta razón deberá estar basada en los valores potenciales de la investigación, viabilidad, así como en la (s) consecuencia (s). Con relación a los valores potenciales no necesariamente deberán incluirse todos, pero sí tendrían que considerarse los siguientes:

1. Conveniencia: para qué sirve, vale o interesa lo que se pretende realizar

2. Relevancia social, económica, ambiental, normativa, científica o tecnológi- 
ca: de qué modo la sociedad se beneficiará (n) con el resultado (s).

3. Implicaciones prácticas: contribuirá a resolver algún problema en el orden práctico o se resolverá otros tipos de problemas a partir de la ejecución práctica

4. Valor teórico: podría contribuir a la generalización de los resultados o aportará sobre algún desconocimiento.

5. Utilidad metodológica: beneficiará a la definición de conceptos, variables o relación entre variables. Sugiere cómo estudiar adecuadamente un campo de acción dentro del objeto de investigación.

\section{- Marco teórico}

En el marco teórico, debe delimitarse qué se ejecutará como investigación a realizarse. Se debe tomar en cuenta el conocimiento previamente construido, pues esta forma parte de una estructura lógica, ya existente y es lo que se denomina marco de referencia (solo se anuncia lo que se hizo por los autores). De forma conjugada, deberá describirse elementos teóricos ya planteados por uno y/o diferentes autores; y que permiten al investigador fundamentar su proceso de investigación (es lo que se conoce como marco teórico propiamente dicho). Finalmente, el investigador define y delimita según su criterio y de acuerdo con su marco teórico, conceptos involucrados en las variables de investigación (es lo que se conoce como marco conceptual).

El marco teórico indica el tipo de estudio o investigación a realizar y eso va a depender de dos aspectos: a) de la información existente; y b) de la interpretación y aplicación que el autor desee dar a sus resultados. Por lo general, indistintamente, se mezcla lo que es un estudio con una investigación. Un estudio no es más una acción planificada para comprender $o$ aprender algo, mientras que una inves- tigación, es un proceso que de la misma forma es planificado pero está destinado al descubrimiento de conocimientos para determinadas soluciones.

\section{- Antecedentes}

En muchos formatos de perfiles de tesis de investigación, consideran antecedentes como un aspecto metodológico independiente al marco teórico, pudiendo ser interpretado como una incoherencia, ya que los antecedentes de estudio relacionados con lo que se pretende estudiar o investigar, está definido como marco referencial o marco teórico.

\section{- Análisis del contexto social}

El análisis del contexto social obedece a una estructura metodológica de enfoque cualitativo donde deberá describirse el espacio o entorno (pueden ser físicos o simbólicos: no son más que los factores culturales, económicos, políticos, históricos, etc.) que sirve de marco para mencionar o entender un determinado episodio, caso o fenómeno. El contexto se crea en base a una serie de circunstancias que ayudan a comprender una información. Las circunstancias para la metodología de la investigación científica deberán ser, según el caso o situación de tipo concreta (s), no abstracta (s). Se observa que es diferente al objeto de investigación.

\section{- Hipótesis}

En muchos formatos de perfiles de tesis de investigación, aparecen hipótesis general e hipótesis específicas, donde puede interpretarse que las suposiciones o conjeturas se elaboran para cada una de las hipótesis específicas están determinadas para cada variable y esto podría ser un error.

Asimismo, habrá que recordar que las hipótesis no son afirmaciones basadas en informaciones donde la relación de las va- 
riables debe ser clara y verosímil. Deben estar relacionadas con técnicas disponibles o que se puedan desarrollar.

Las hipótesis surgen en el planteamiento del problema científico una vez que ha sido reevaluado, después del marco teórico.

En las investigaciones cuantitativas, las hipótesis pueden clasificarse de forma general en cuatro tipos. A su vez, la hipótesis de investigación como la hipótesis estadística puede ser clasificada en otros tipos de la siguiente manera:

1. Hipótesis de investigación: descriptiva, correlacional, diferencias entre grupos y relaciones de causalidad.

2. Hipótesis nula: son el reverso de la hipótesis de investigación.

3. Hipótesis de alternativa: adquieren una posición intermedia entre la hipótesis de investigación y la hipótesis nula.

4. Hipótesis estadística: de estimación, de correlación, de diferencias entre medias.

Para el caso de las investigaciones cualitativas, no necesariamente existen hipótesis. Sin embargo, parece más evidente que estas puedan ser definidas cuando se aplica el método de teoría fundamentada, pues cuando se hace la teoría (tercer orden en la construcción del silogismo), estas pueden ser de dos tipos, teoría sustantiva y teoría formal, entonces posibilitan anunciar preferiblemente las hipótesis.

\section{- Objetivo}

Los objetivos pueden ser general (es) que contribuyen a la solución del problema científico y específico (s), los cuales permitan hallar los subproblemas encontrados. Cuando se tienen objetivos específicos, tienen que ser sucedáneos, ya que de lo contrario no tiene sentido que se redacten. Existe un dogmatismo como obstáculo en la ciencia que debe existir un objetivo general y tres específicos, siendo esto incorrecto, ya que en cualquier tipo de estudio como investigación, puede existir más de un objetivo general, así como menos o más de tres. Los objetivos se redactan en infinitivo verbal, pero sin cargarlo de subjetividad y están compuestos por una acción, producto y resultado. Existe una clasificación de los objetivos, y están comprendidos en textos sobre metodología de la investigación científica, por ejemplo, en el trabajo de Romey (1969). Lo significativo es interpretar la clasificación, atendiendo al tipo de estudio o investigación a realizar.

\section{- Materiales y métodos}

Por lo general, en los materiales y métodos se consideran los aspectos relacionados con el objeto de investigación y periodo de estudio, población y muestra, análisis de variables, análisis de los datos y la operacionalización de las variables, siendo requeridas, en cada aspecto, las informaciones siguientes:

1. Objeto de investigación

- Descripción geográfica de la zona de estudio

- Importancia de la selección de la zona de estudio

2. Población y muestra

- Clasificación de la población (finita o infinita)

- Selección de la muestra, mencionando de acuerdo con su clasificación si es probabilística o no probabilística.

- Condiciones de muestreo (equipos y/o utensilios, frecuencia de muestreo, conservación de muestras)

- Indicar el criterio de fórmula utilizado para la representación significativa de la muestra.

3. Descripción analítica del o los métodos por objetivos, señalando su referencia 
- Indicar las variables objeto de investigación según el tipo de investigación: cualitativa (nominal u ordinal), cuantitativa (discreta o continua; dependiente e independiente, confusoras /intervinientes).

4. Descripción o citación de las técnicas analíticas a utilizar, señalando su referencia.

5. Análisis de los datos

- Paquete estadístico a emplear

- Estadígrafo (s) paramétrico (s) o no paramétrico (s) a utilizar

- Nivel de significación o confianza de los datos

6. Operacionalización de las variables
La tabla de operacionalización de las variables presenta seis columnas estrechamente relacionadas. En muchos de los formatos de investigación, se establece como razón obligatoria la identificación de variables independientes y dependientes, siendo ello un error, pues lo único que se está reflejando es que el estudio será explicativo o correlacional, ya que son los que justifican el uso de este tipo de variable clasificada, informa, además, que el enfoque a realizarse será cuantitativo, debido a que en los estudios e investigaciones cualitativas este tipo de variable no está denominada.

La tabla de operacionalización de las variables puede quedar determinada de la siguiente manera:

\begin{tabular}{|l|l|l|l|l|l|}
\hline Variable & Dimensión & Indicador & Categoría & Índice & Instrumento \\
\hline & & & & & \\
\hline & & & & & \\
\hline & & & & & \\
\hline
\end{tabular}

a. Variable. Propiedad medible dada su capacidad de variación

b. Dimensión. Capacidad de extensión relacionada con la variable de selección

c. Indicador. Lo que indica la expresión de la variable seleccionada

d. Categoría. Tipo de variable seleccionada en forma clasificada

e. Índice. Unidad de medida del indicador

f. Instrumento. Con lo que se mide la variable seleccionada

- Presentación de tabla (s) de construcción de caso (s)

La presentación de tabla (s) de construcción de caso (s), solo es considerada para los estudios o investigaciones cualitativas, donde se deberá elaborar el cómo indicar la observación del (los) factor (es) o variable (s).

\section{- Cronograma de actividades}

Generalmente, no se detalla lo que se realizará desde el nivel general hasta el específico y donde muchas tablas de cronograma de actividades deberán expresar con claridad la planificación temporal sobre la ejecución. La tabla de cronograma de actividades no es más que un instrumento de aprendizaje que refleja un esquema numérico.

La tabla de cronograma de actividades puede quedar determinada de la siguiente manera:

\begin{tabular}{|c|c|c|}
\hline Cronograma & \multicolumn{1}{|c|}{ Indicar planificación / meses } \\
\hline 1. Actividad & & \\
\hline 1.1 Tarea & & \\
\hline 1.1 .1 Acción & & \\
\hline
\end{tabular}


a. Actividad. Facultad de obrar con prontitud los objetivos específicos.

b. Tarea. Labor a realizar en un tiempo limitado.

C. Acción. Es el valor que acredita con cuánto se ha contribuido en el tiempo.

\section{- Presupuesto de la investigación}

En muchos formatos de perfil de tesis, se presentan informaciones con cierto grado de ambigüedad, además de la falta de control y utilización de los recursos, pues en muchas de las propuestas de tesis, las mismas no están basadas sobre un proyecto propiamente dicho como instrumento gerencial operativo que concentra recursos para dar solución a un desafío del entorno social que es complejo y que tiene una meta o fin, por cuanto en reiteradas ocasiones queda a informaciones plasmadas incorrectas.

La tabla de presupuesto de la investigación puede quedar determinada de la siguiente manera

\begin{tabular}{|l|l|l|l|l|}
\hline $\begin{array}{c}\text { Rubro } \\
\text { (descripción) }\end{array}$ & Cantidad & $\begin{array}{c}\text { Unidad de } \\
\text { Medida }\end{array}$ & $\begin{array}{c}\text { Costo } \\
\text { Unitario }\end{array}$ & Costo Total \\
\hline Recursos humanos & & & & \\
\hline Recursos materiales & & & & \\
\hline Recursos financieros & & & & \\
\hline
\end{tabular}

\section{- Referencias}

Se considerarán fuentes de información primaria, secundaria y terciaria. Asimismo, el formato variará según el criterio de cada institución, donde deberá considerarse sus carreras profesionales de formación, pues ello indicará, por ende, el tipo de estilo para la orientación hacia determinadas revistas indexadas, al menos, en bases de datos de los grupos I y II con alto prestigio internacional.

\section{Discusión}

Resulta muy relevante entender con claridad que significa el silogismo deductivo e inductivo que caracteriza a las investigaciones con enfoque cuantitativo y cualitativo, respectivamente. Las Figuras 1 y 2 , informan lo que ello representa.

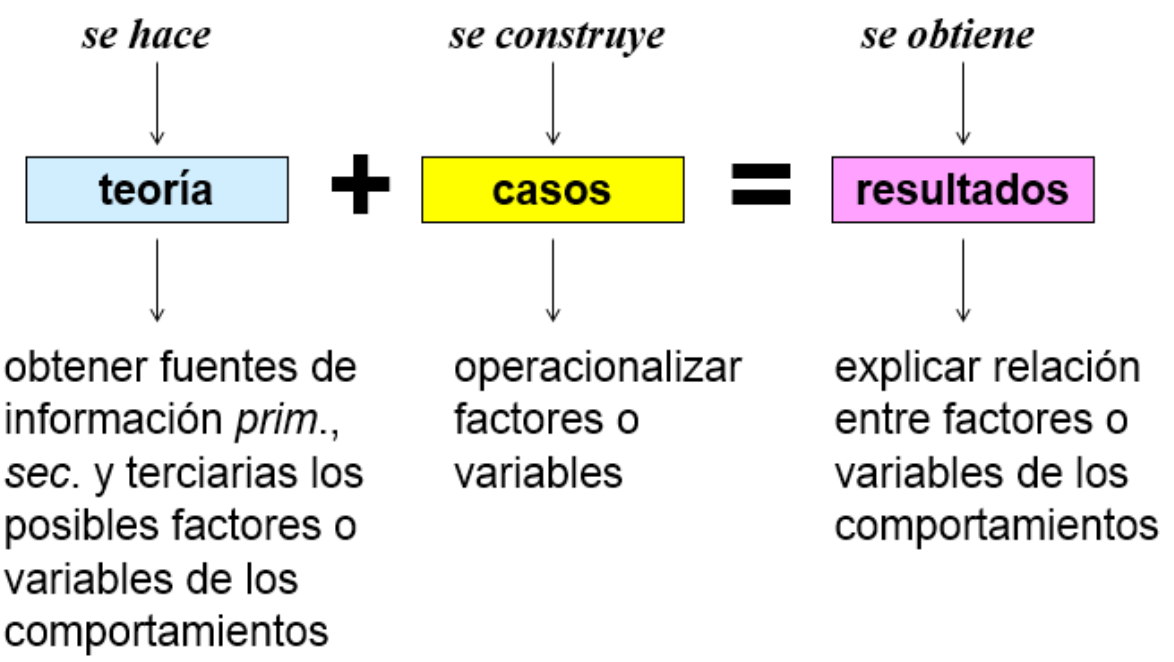

Figura 1. Silogismo deductivo (enfoque cuantitativo) 


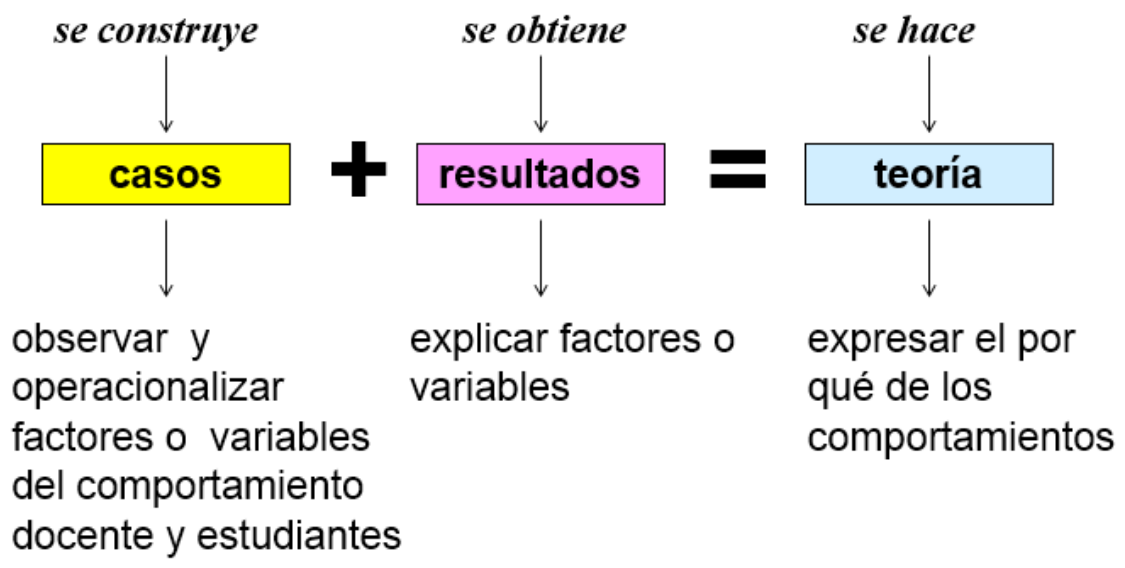

Figura 2. Silogismo inductivo (enfoque cualitativo)

Las figuras anteriores demuestran que una investigación puede comenzar siendo cuantitativa y terminar siendo cualitativa y de forma viceversa, por lo que si ello es así, entonces sería necesario que se tenga en cuenta que ello puede ser posible y por tanto, todo investigador no puede estar influenciado por su enfoque de formación, ya que la única consecuencia será poder enfrentar de forma limitada determinadas variables dentro de una causa y no reconocer al problema científico como un estado actual no deseado que es presentado de forma multicausal.

Jick (1979) introdujo los términos básicos de los diseños mixtos, al recurrir a técnicas e instrumentos proporcionados por paradigmas positivistas y naturalistas para la recolección de datos, dando un lugar prioritario a la triangulación de datos, de manera que las informaciones descritas, en cada estructura metodológica, posibilita dicha triangulación.

Los conocimientos desarrollados a lo largo del tiempo, permitieron señalar las diferentes características y modalidades que dependiendo del objeto de estudio, podrían adoptar los diseńos mixtos; y en ese sentido, autores como Creswell, Plano, Gutmann y Hanson (2003), denominaron los diseños mixtos como el tercer movimiento metodológico. Johnson y Onwuegbuzie (2004) definieron los diseños mixtos como el tipo de estudio donde el investigador mezcla o combina técnicas de investigación, métodos, enfoques, conceptos o lenguaje cuantitativo o cualitativo en un solo estudio.

Rocco, Bliss, Gallagher y Pérez (2003) argumentan que los diseños mixtos se fundamentaron en la posición pragmática (el significado, valor o veracidad de una expresión se determina por las experiencias o las consecuencias prácticas que tiene en el mundo) o en la posición dialéctica (hay una mejor comprensión del fenómeno cuando se combinan los paradigmas) y conformaron, así una tercera fuerza en la investigación.

Para Driessnack, Sousa y Costa (2007), los métodos mixtos se refieren a un único estudio que utiliza estrategias múltiples o mixtas para responder a las preguntas de investigación y/o comprobar hipótesis. Si bien es cierto que las investigaciones con enfoque cualitativo no parten sobre la demostración de hipótesis, pero tiene que tenerse en cuenta que el método cualitativo por teoría fundamentada puede generar hipótesis y con esta, enfrentar, seguidamente, una investigación cuantitativa, por lo que las informaciones descritas, pueden interpretarse para realizarlas.

Christ (2007) argumenta que la investigación mediante métodos mixtos se ha for- 
talecido en los últimos veinte años y los estudios exploratorios cualitativos, seguidos de estudios confirmatorios, han sido comunes y concurrentes, por lo que se sigue demostrando que solo enfrentar las investigaciones sobre una determinada posición, quizás solo podría mitigar al problema científico en un porcentaje bajo, de manera que Dellinger y Leech (2007), igualmente analizan y corroboran la validez de los métodos mixtos en la investigación científica.

Para Cameron (2009), la investigación con métodos mixtos ha ido generando cambios metodológicos entre los investigadores y académicos en una variedad de áreas disciplinarias. Esta información demuestra que los formatos de investigación presentados por cualquier universidad, no pueden estar sujetos a un tipo de investigación, ya que no es posible que la formación influenciada por un determinado enfoque y finalmente, al término de una graduación, entonces haya que enfrentar otros tipos de paradigmas.

Se concluyó que la descripción interpretativa para la elaboración del perfil de tesis de investigación científica con enfoque mixto resultó de gran beneficio, pues ello permitió que se pueda dimensionar el patrón de comportamiento sobre el fenómeno, suceso, proceso o evento observado y donde es susceptible, finalmente a investigar.

\section{Referencias}

Argota, G. \& Iannacone, J. (2015). Significación de formatos metodológicos como guías de aprendizaje para la investigación científica de postgrado en ciencias naturales y no afines de universidades peruanas. The Biologist, 13(1), 41-51. Recuperado de http:// sisbib.unmsm.edu.pe/bvrevistas/biologist/v13_n1/pdf/a05v13n1.pdf

Artigas, W \& Robles, M. (2010). Metodología de la investigación: Una discusión necesaria en Universidades. Revista Digital Universitaria, 11: 16 pp. Recuperado de http://www.revista.unam. $\mathrm{mx} /$ vol.11/num11/art107/art107.pdf

Barrientos, J.C., Rubio, B.P., Garza, F.R., Rolón, J.C., Laria, J., Rodríguez, Á. \& Vázquez, J. M. (2007). Guía para la presentación del informe de investigación científica. Recuperado de http:// www.geiuma-oax.net/seminario/reportede investigacion.pdf

Cameron, R. (2009). The use of mixed methods research in VET research. Proceedings of aligning participant's policy and pedagogy: traction and tension in VET research: 12th AVETRA Annual Conference, Sydney, NSW. Recuperado de http://epubs.scu.edu.au/cgi/ viewconten .cgi?aticle $=1158 \&$ contex $=$ comm_pubs

Charaja, CF. (2011). La ciencia como proceso. Episteme, 1: pp 23-37.

Christ, T. (2007). A Recursive Approach to Mixed Methods Research in a Longitudinal Study of Postsecondary Education Disability Support Services. Journal of Mixed Methods Research, 1(3), 226-241.

Cornejo, M. \& Salas, N. (2011). Rigor y calidad metodológicos: un reto a la investigación social cualitativa. Psicoperspectivas. Individuo y Sociedad, 10(2), 12-34. Recuperado de http://www. scielo.cl/pdf/psicop/v10n2/art02.pdf

Creswell, J. W., Plano, C. V. L., Gutmann, M. L. \& Hanson, W. E. (2003). Advanced mixed methods research designs. En A. Tashakkori \& C. Teddlie (Eds.), Handbook of mixed methods in 
social and behavioral research (pp. 209240). Thousand Oaks, CA: Sage.

Dellinger, A. \& Leech, N. (2007). Toward a Unified Validation Framework in Mixed Methods Research. Journal of Mixed Methods Research, 1(4), 309-332.

Driessnack, M., Sousa, V. \& Costa, I. (2007). Revisión de los diseños de investigación relevantes para la enfermería: parte 3: métodos mixtos y múltiples. Revista LatinoAmericana de Enfermagem, 15(5), 179-182. Recuperado de http://www.scielo.br/pdf/rlae/v15n4/ es_v15n4a25.pdf

Grinell, R. (1997). Social work research \& evaluation: Quantitative and qualitative approaches ( $5^{\text {th }}$ ed.). Illinois: E.E. Peacock Publishers.

Hernández, R., Fernández, C. \& Baptista, P. (2003). Metodología de la Investigación (3 $3^{a}$ ed.). México: McGraw Hill.

Jick, T. D. (1979). Mixing qualitative and quantitative methods: Triangulation in action. Administrative Science Quarterly, 24, 602-611. Recuperado de http://citeseerx.ist.psu.edu/viewdoc/ downad?doi=10.1.1.317.1797\&re$\mathrm{p}=$ rep $1 \&$ type $=$ pdf

Johnson, B. \& Onwuegbuzie, A. (2004). Mixed Methods Research: A Research Paradigm Whose Time Has Come. Educational Researcher, 33(7), 14-26. Recuperado de http://www.tc.umn.edu/ dillon/CI\%208148\%20Qual\%Reserch/Seccion\%2014/Johnson\%20 \&\%Onwuegbuxie\%20PDF.pdf

Miles, M. \& Huberman, A. (1994). Qualitative Data Analisis (2 ${ }^{\text {nd }}$ ed.). New York: Sage Publications.

Namakforoosh, M.N. (2005). Metodología de la Investigación (2a ed.). México D.F.: Editorial Limusa.
Okuda, B.M. \& Gómez, R.C. (2005). Métodos en investigación cualitativa: triangulación. Revista Colombiana de Psiquiatría; 34(1), 118-124.

Ortiz, F. \& García, Ma.P. (2005). Metodología de la Investigación. México D.F: Editorial Limusa.

Rocco, T., Bliss, L., Gallagher, S. \& Pérez, P. A. (2003). Taking the Next Step: Mixed Methods Research in Organizacional Systems. Information Technology, Learning, and Performance Journal, 21(1), 19-29. Recuperado de http://citeseerx.ist.psu.edu/viewdoc/ down?doi=10.1.1.122.1050\&rep=rep1\&type $=$ pdf

Romey, W. D. (1969). Inquiry techniques for teaching science. Englewood Cliffs: Prentice-Hill.

Ruiz, M.I., Borboa, M. S. \& Rodríguez, J. C. (2013). El enfoque mixto de investigación en los estudios fiscales. Revista Académica de Investigación TLATEMOANI, 13, 1-25. Recuperado de http://www.eumed.net/rev/tlatemoani/13/estudios-fiscales.pdf

Ruiz, R. (2007). El método cientifico y sus etapas. Recuperado de http://www. index-f.com/lascasas/documentos/ lc0256.pdf

Stoecker, R. (1991). Evaluating and rethinking the case study. Social Review, 39(1), 88-112.

Vara, A. A. (2012). Desde la idea hasta la sustentación: siete pasos para una tesis exitosa. Un método efectivo para las ciencias empresariales [versión PDF]. Recuperado de http://www.administracion. usmp.edi.pe/institutoinvestigacion/ wp-content/uploads/sites/9/2014/02/ Manual_7passos_aristidesvara.pdf 